\title{
Stress determination of micromembranes using laser vibrometry
}

\author{
Abhijit Biswas ${ }^{a)}$ \\ MS 183-401, Advanced Materials and Fluid Processes Technology Group, Jet Propulsion Laboratory, \\ California Institute of Technology, Pasadena, California 91109 \\ Tom Weller ${ }^{\text {b) }}$ \\ Microwave \& Wireless Research Group, Electrical Engineering Department, University of South Florida, \\ ENB 118, Tampa, Florida 33620 \\ Linda P. B. Katehic) \\ Radiation Laboratory, University of Michigan, 3228 EECS Building, Ann Arbor, Michigan 48109-2122
}

(Received 29 November 1995; accepted for publication 19 February 1996)

\begin{abstract}
A laser vibrometry based dynamic technique for biaxial residual stress determination in micromembranes is reported. Layered, low pressure chemical vapor deposition grown, rectangular shaped micromembranes are ultrasonically excited. The resulting micromembrane displacement, as a function of frequency and position, is recorded to yield resonance frequencies and associated vibrational mode numbers. The stress is determined from this information. Vibrations forced from ultrasonic pressure $(\sim 25 \mathrm{~Pa})$ resulted in peak displacements of tens of nanometers at resonance. For the sample set studied, the $(3,1)$ mode resonance peak displayed the least damping in atmospheric testing and was used to establish stress levels $\sim 10^{8} \mathrm{~N} / \mathrm{m}^{2}$. A trend for this stress showing an overall decrease by $\sim 40 \%$ as a result of thermomechanical cycling was identified. (C) 1996 American Institute of Physics. [S0034-6748(96)05105-2]
\end{abstract}

\section{INTRODUCTION}

The emergence of micromembrane-based architectures has resulted in a need for accurate analysis of membrane stress characteristics. Micromembranes are increasingly being used for diverse applications such as $\mathrm{x}$-ray lithography masks ${ }^{1-4}$ and microwave transmission lines. Examples of membrane-based microwave components include switches, very high- $Q$ inductors, $K \alpha$-band power dividers, and filters operating from 15 to $250 \mathrm{GHz}^{5-9}$ In order to successfully implement these structures in practical devices, it is important to investigate the residual biaxial stress build-up during fabrication, and the subsequent evolution of the stress with thermomechanical cycling. This knowledge is critical if one is to resolve and correct for stress-related failure mechanisms. Electronic components using membrane technology are increasingly being envisaged for miniaturization of future space flight hardware. Here, too, mechanical property dependence on structural vibrations and thermal cycling is crucial for reliable implementation.

Previous work on membrane stress characterization includes both theoretical and experimental methods. Analytical and finite element stress analysis of homogeneous, rectangular, and circular membranes ${ }^{10,11}$ can predict the biaxial stress and Young's modulus of membranes. Studies of stress and Young's modulus by static (load versus deflection) measurement methods have also been reported. ${ }^{11,12}$ In addition, dynamic techniques have been used where the resonant frequencies of vibrating membranes are used (as elaborated below) for determining stress. ${ }^{1,13-15}$

A new dynamic technique for membrane stress determi-

\footnotetext{
${ }^{a)}$ Electronic mail: abhijit biswas@jpl.nasa.gov

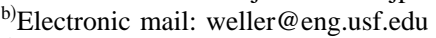

${ }^{c)}$ Electronic mail: katehi@eecs.umich.edu
}

nation utilizing laser vibrometry is described in the present article. Specifically, a tightly focused laser spot is used for interrogating the driven (vibrating) membrane surface while extracting displacement and velocity spot measurements from an interferometric analysis of the retroreflection. The technique allows a comprehensive characterization of vibrating micromembranes and can potentially accommodate nonstandard geometries, layered micromembrane architectures, and a variety of in situ monitoring scenarios where evolution of stress is of interest.

Based on membrane vibration theory ${ }^{16,17}$ the driven rectangular membrane exhibits resonances at frequencies $f_{m n}$ given by the relation:

$$
f_{m n}=\frac{1}{2} \sqrt{\frac{\sigma}{\rho}}\left(\frac{m^{2}}{l x^{2}}+\frac{n^{2}}{l y^{2}}\right)^{0.5},
$$

where $\sigma$ and $\rho$ are biaxial stress and density, while $l x$ and $l y$ represent rectangular membrane dimensions. A frequency sweep with the laser vibrometer while recording displacement yields $f_{m n}$, while modal analysis of individual peaks identifies $m$ and $n$. Knowing $l x, l y, m, n$, and $\rho$ allows a reliable determination of the stress, $\sigma$. Equation (1) holds for ideal membranes and, as elaborated in Ref. 15, several factors, such as atmospheric loading, contributions from bending stiffness, and an anisotropic state of stress, contribute to deviations from ideal membrane behavior.

Atmospheric loading causes resonant frequencies measured in open air to be lowered relative to values obtained in vacuum. A suggested correction ${ }^{1}$ to properly account for this is given below:

$$
f_{m n}=\left(f_{m n}\right)_{\mathrm{air}}\left[1.34\left(\frac{L \rho_{\mathrm{air}}}{t \rho}\right)\right],
$$




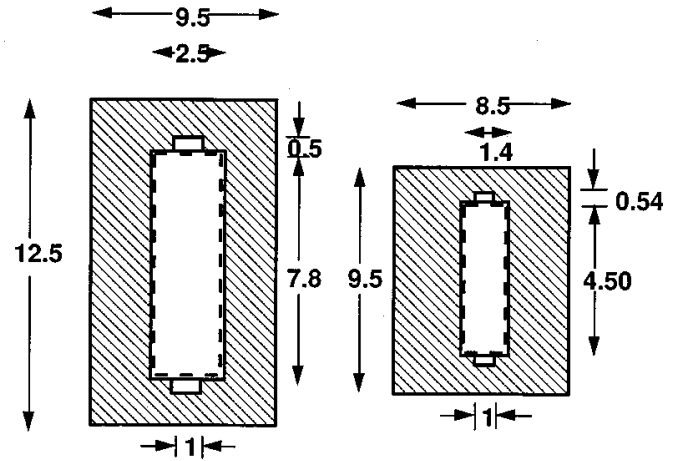

FIG. 1. Schematic view of the membranes, with dimensions in $\mathrm{mm}$, supported by a Si substrate (hatched region). The membrane outline is shown by dashed lines while the metallization outline is shown by dark lines. Details of the gold pattern overlay covering $95 \%$ of the membranes are not shown.

where $L$ is defined as $\sqrt{ }(l x . l y), t$ is membrane thickness, and $\rho_{\text {air }}$ is air density. The stiffness effect can be neglected if

$$
\frac{D \pi^{2}}{\sigma l_{x} l_{y}} \ll 1, \quad \text { where } \quad D=\frac{E t^{2}}{12\left(1-\nu^{2}\right)},
$$

$E$ being the Young's modulus and $\nu$ the Poisson's ratio of the membrane material.

\section{EXPERIMENT}

The type of micromembrane examined in this study consists of a thin-film coplanar waveguide (CPW) transmission line which is supported on a three-layer dielectric membrane. The membrane is grown on a silicon wafer and comprises a high temperature oxide (HTO) and subsequent silicon nitride and oxide layers. Each of the top layers is deposited using low pressure chemical vapor deposition (LPCVD). By properly balancing the thickness of the three layers, a composite in slight tension is obtained that remains rigid when portions of the silicon substrate are removed. High-yield membranes are obtained on 10-cm-diam, 500-550- $\mu$ m-thick wafers using thicknesses of 750/350/450 $\mathrm{nm}$ for the thermal oxide, intermediate nitride, and top oxide layers, respectively. Gold metallization 1 to $1.2 \mu \mathrm{m}$ thick covers $95 \%$ of the membrane surface. Figure 1 shows the shape and dimensions of micromembranes used in the present study.

Three sets of micromembranes with different thermomechanical histories, as elaborated in Table I, were tested. Random vibration testing for $180 \mathrm{~s}$ durations were conducted along the three principle axes of the membranes. Vibration frequencies ranged from $20-2000 \mathrm{~Hz}$ and levels up to 0.8 $\mathrm{g}^{2} / \mathrm{Hz}$ were used. Thermal cycle time was $1 \mathrm{~h}$.

Dynamic testing of the micromembranes was carried out at atmospheric pressure using the experimental arrangement

TABLE I. The thermomechanical history of the sample set used for performing stress measurements.

\begin{tabular}{lc}
\hline \hline Set 1 & As received \\
Set 2 & Vibration tested, 100 thermal cycles (208 to $423 \mathrm{~K})$ \\
Set 3 & 100 thermal cycles (208 to $423 \mathrm{~K})$, vibration testing, \\
& 100 thermal cycles (208 to 423 K) \\
\hline \hline
\end{tabular}

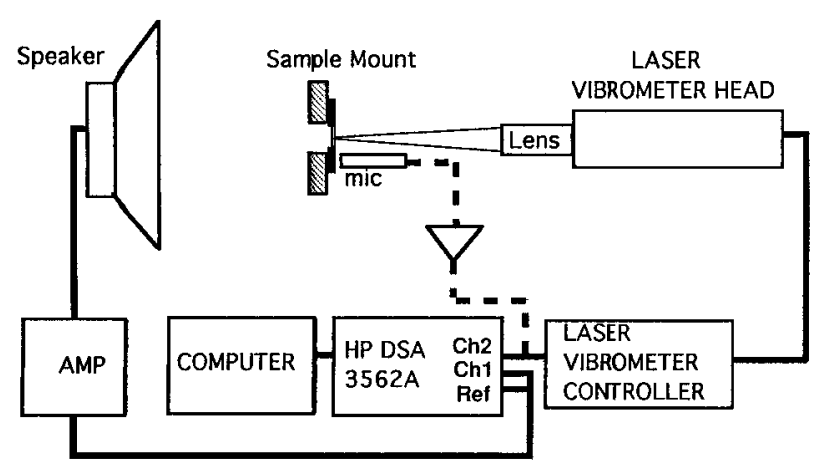

FIG. 2. A schematic layout of the experimental arrangement used to perform the vibrational analysis of the membranes.

shown in Fig. 2. Samples were mounted on a rigid support using double sided adhesive, with the focused spot from the laser vibrometer incident on the micromembrane. Microtranslation of the micromembrane with respect to the incident laser spot was possible. The sample mount, laser vibrometer, and audio speaker were supported on an air table to minimize noise from extraneous vibrations. Pressure fluctuations generated by the audio speaker (JBL Model 4370) excited vibrations over a frequency range $15-60 \mathrm{kHz}$.

The laser vibrometer manufactured by Polytec PI Inc. (Model 0FV-302) utilizes an integrated Mach-Zender interferometer. Separate displacement and velocity mode outputs
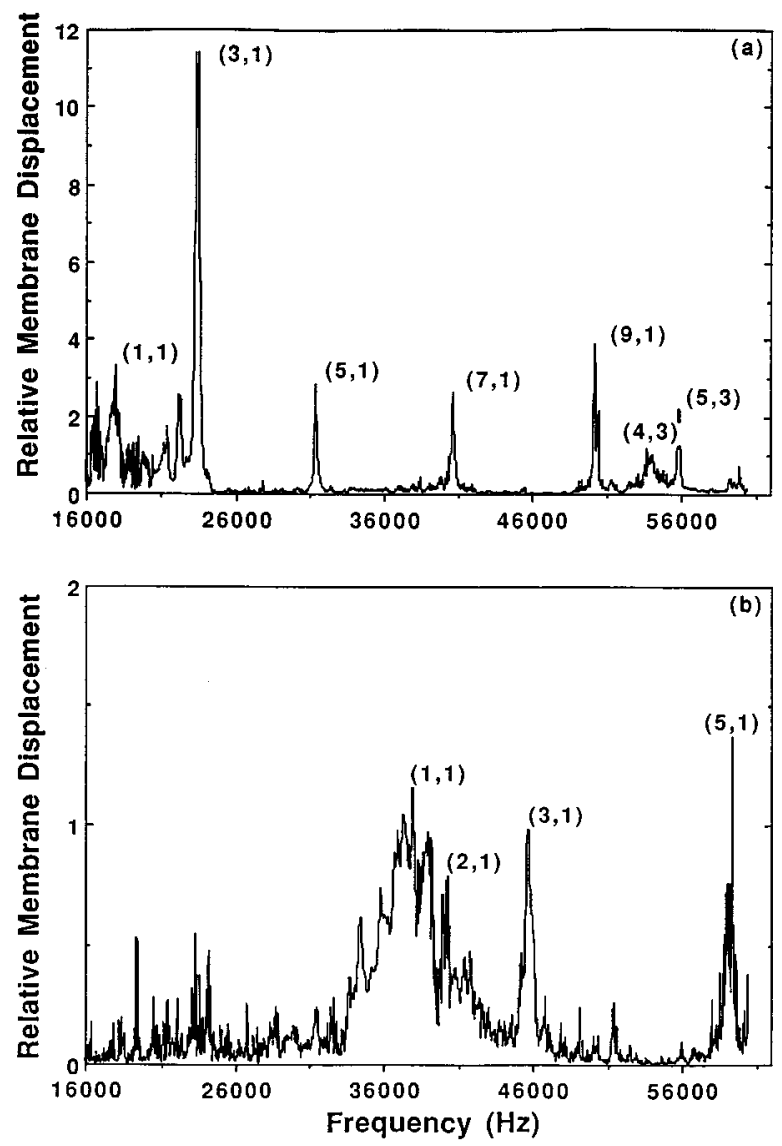

FIG. 3. (a) Displacement spectrum of a large membrane sample $(7.8 \times 2.5$ $\mathrm{mm}$, sample set 3). (b) Displacement spectrum of a small membrane sample $(5.04 \times 1.2 \mathrm{~mm}$, sample set 3$)$. 


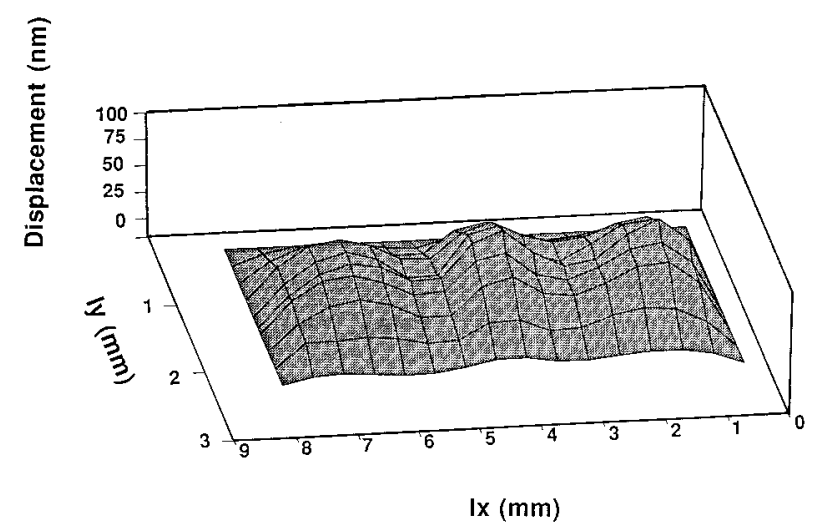

FIG. 4. Surface plot showing the spatial variation of displacement for a $(3,1)$ resonance.

are obtained after appropriate processing of the optical interference signal derived from the retroreflection. The corroboration of results using the two modes was checked and found to be excellent, although the displacement mode was used for all the measurements. Absolute displacement and velocity determination relies on instrument constants. The reliability of the conversion constant was verified for displacements ranging from $100 \mathrm{~nm}-2 \mu \mathrm{m}$ at $500 \mathrm{~Hz}$, using a magnetostrictive device where the current versus displacement was known. The displacement sensing bandwidth is $1 \mathrm{MHz}$ and flat in the region of interest. The focused laser spot size was measured using the knife-edge method and found to be nominally $40 \mu \mathrm{m}$ FWHM.

A digital signal analyzer (DSA) (HP Model 3562A) was used to perform a linear sine sweep (resolution $25 \mathrm{~Hz}$ ) of the micromembrane response. A sinusoidal source signal with a fixed amplitude (100 $\mathrm{mV}$ peak to peak), from the DSA, is simultaneously applied to channel 1 of the DSA and the audio amplifier driving the speaker. The laser vibrometer analog displacement output is fed to channel 2 of the DSA and the ratio of power spectra (PS channel 1 to PS channel 2) is recorded. PS channel 1 is flat since it originates from a fixed amplitude sinusoidal signal thereby serving as a reliable reference. Variations in PS channel 2 represent relative membrane displacement as a function of frequency. Identical frequency sweeps performed with a $1 / 4$ in. microphone (Bruel and Kjaer, Model 4135) positioned at the location of the membrane were used to correct for frequency dependent variations in audio amplifier gain and speaker output. The microphone response allowed an estimate of the peak pressure driving the micromembranes, and this was $\sim 25 \mathrm{~Pa}$.

Both membrane and microphone response were verified to be linear for the speaker output levels used. The laser vibrometer was unable to sense any displacement from regions of the substrate along the boundary ensuring that sig-

TABLE II. A summary of observed frequency peaks, mode assignments, percentage deviations between expected and observed peak positions, and derived biaxial stress for the entire sample set studied.

\begin{tabular}{|c|c|c|c|c|}
\hline Sample & $\begin{array}{l}\text { Observed } \\
\text { frequency } \\
\quad(\mathrm{Hz})\end{array}$ & $\begin{array}{l}\text { Mode } \\
\text { number }\end{array}$ & $\%$ deviation & $\begin{array}{l}\text { Stress } \\
\left(\mathrm{N} / \mathrm{m}^{2}\right)\end{array}$ \\
\hline $7.8 \times 2.5 \mathrm{~mm}$ & 23333 & $(1,1)$ & 1.7 & \\
\hline \multirow[t]{4}{*}{ Set 1} & 26032 & $(2,1)$ & 3 & \\
\hline & 31349 & $(3,1)$ & $\ldots$ & \\
\hline & 42460 & $(5,1)$ & 0.5 & $2.13 \times 10^{8}$ \\
\hline & 55159 & $(7,1)$ & 0.7 & \\
\hline $5.04 \times 1.2$ & 41746 & $(1,1)$ & 0.7 & \\
\hline Set 1 & 50317 & $(3,1)$ & $\cdots$ & $1.78 \times 10^{8}$ \\
\hline $7.8 \times 2.5 \mathrm{~mm}$ & 23452 & $(1,1)$ & 4.7 & \\
\hline \multirow[t]{4}{*}{ Set 2} & 26349 & $(2,1)$ & 4 & \\
\hline & 29523 & $(3,1)$ & $\cdots$ & \\
\hline & 40238 & $(5,1)$ & 0 & \\
\hline & 52460 & $(7,1)$ & 0.3 & $1.89 \times 10^{8}$ \\
\hline $5.04 \times 1.2 \mathrm{~nm}$ & 50159 & $(1,1)$ & 1.7 & \\
\hline Set 2 & 58968 & $(3,1)$ & $\ldots$ & $1.80 \times 10^{8}$ \\
\hline $7.8 \times 2.5 \mathrm{~mm}$ & 17780 & $(1,1)$ & 1.4 & \\
\hline \multirow{6}{*}{ Set 3} & 23161 & $(3,1)$ & $\ldots$ & \\
\hline & 31323 & $(5,1)$ & 0.7 & \\
\hline & 40471 & $(7,1)$ & 1.35 & \\
\hline & 50157 & $(9,1)$ & 1.6 & \\
\hline & 54013 & $(4,3)$ & 0.8 & \\
\hline & 55807 & $(5,3)$ & 1.6 & $1.16 \times 10^{8}$ \\
\hline $5.04 \times 1.3 \mathrm{~mm}$ & 37242 & $(1,1)$ & 2.1 & \\
\hline \multirow[t]{3}{*}{ Set 3} & 40022 & $(2,1)$ & 2.4 & \\
\hline & 45493 & $(3,1)$ & $\ldots$ & \\
\hline & 59036 & $(5,1)$ & 2.5 & $1.07 \times 10^{8}$ \\
\hline
\end{tabular}




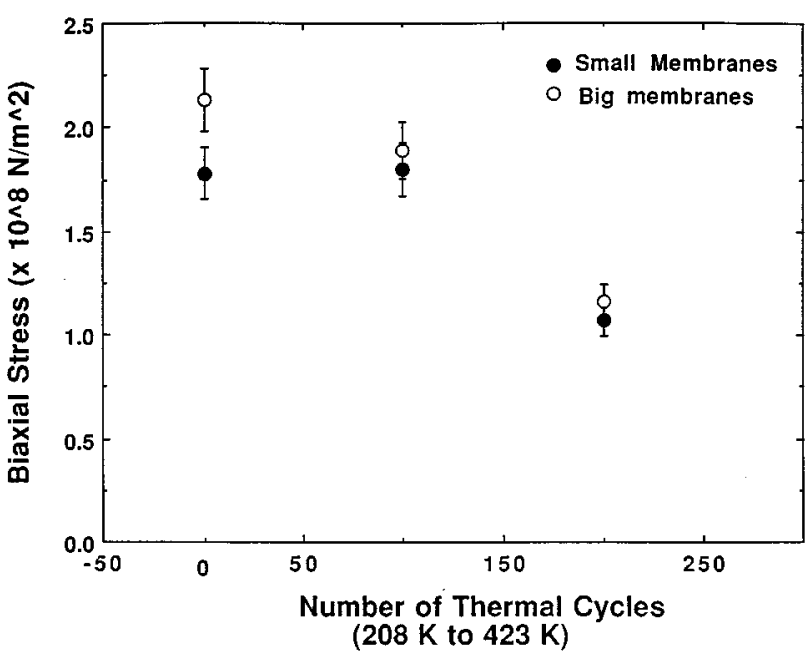

FIG. 5. Plot showing the evolution of stress as a function of thermal cycles. Vibration testing was also performed on the samples (see Table I).

nificant translational motion of the substrate/membrane assembly did not occur. Laser vibrometer output recorded in the absence of excitation indicated that the noise floor was 2-3 orders of magnitude below the membrane driven response.

\section{RESULTS}

Figure 3 shows the swept sine response for a "big" and "small" membrane (see Fig. 1). Resonance peaks are shifted to higher frequencies for the smaller dimensioned membranes, as expected. The presence of an initial broad peak followed by sharper peaks was typical of all the samples tested.

A modal analysis of the first sharp, well-defined peak was undertaken by translating the micromembrane with respect to the focused laser spot, while driving the membrane at the resonance frequency. The peak of the averaged power spectrum, measured by the DSA, was converted to membrane displacement. The results, shown as the surface plot of Fig. 4, clearly indicate an $m=3, n=1$ mode. Maximum displacements of $80 \mathrm{~nm}$ and minimum displacements of $3 \mathrm{~nm}$ were detected. Reliability of these absolute numbers is subject to the conversion constants discussed above. The observed displacements could not be verified by computation since this would entail a knowledge of the damping forces operative and ascertaining this parameter was beyond the scope of the present study.

Micromembrane dimensions (see Fig. 1) of $l x=7.8 \mathrm{~mm}$, $l y=2.5 \mathrm{~mm}$, and $l x=5.04 \mathrm{~mm}, l y=1.2 \mathrm{~mm}$ were measured for the big and small samples. A weighted micromembrane density, $\rho=10083 \mathrm{~kg} / \mathrm{m}^{3}$, was calculated based on the relative thickness of $\mathrm{SiO}_{2}, \mathrm{Si}_{3} \mathrm{~N}_{4}$, and $\mathrm{Au}$. Using these values and the peak frequency, $f_{31}$, the stresses for each membrane were calculated. Mode numbers for other observed peaks in the swept sine spectra were compared to calculated values using the method of ratios $f_{m n} / f_{31}$. A complete summary of determined stress using the correction given by Eq. (2) and the accompanying mode assignments along with \% deviation from calculated values is presented in Table II. The modes for the peaks observed in Fig. 3 are also shown.

The dimensions and materials of the membranes used are such that the condition defined by the inequality (3) is satisfied; hence, the effect of stiffness on resonance characteristics can be neglected. A general trend observed in all the measurements is the highly damped and poorly defined nature of the fundamental $(1,1)$ peak. This observation is further exaggerated for the smaller membranes, as shown in Fig. 3. The $(3,1)$ mode, however, appears to be a relatively high quality factor resonance and is better suited for reliable stress determination than the lower order modes. A survey of Table II also shows other inexplicable features, such as (i) the membranes tested display odd numbered modes such as $(5,1)(7,1)$ and $(9,1)$ while corresponding, even $m$, modes do not appear; (ii) the uncertainty in identifying the modes lower than $(3,1)$ is higher than that encountered for higher order modes; and (iii) modes such as $(4,3)$ and $(5,3)$ seem to appear in one of the samples with many intermediate modes being suppressed. Observations of this nature were not found in previous reports.

Finally, Fig. 5 shows a summary of the residual biaxial stress obtained for the entire sample set. The derived stress is displayed as a function of the number of thermal cycles. An overall trend of reduction in stress as a result of thermomechanical processing is observed. The error bars shown were chosen to be $\sim 7 \%$ based on uncertainties in the membrane thickness and dimensions. The conjecture can be made that the combination of mechanical and thermal cycling brings about a lowering of the clamping force at the membrane boundaries due to differential thermal expansion and elastic coefficients between the membrane and substrate.

\section{ACKNOWLEDGMENTS}

The work would not have been possible without the guidance and assistance provided by Marty Herman, Elizabieta Kolawa, and Beverly Tai. This work was partially performed at the Jet Propulsion Laboratory, California Institute of Technology, and was supported jointly by the National Aeronautics and Space Administration and Caltech through the Caltech President's Fund.

${ }^{1}$ M. Karnezos, J. Vac. Sci. Technol. B 4, 226 (1986).

${ }^{2}$ K. Marumoto, H. Yabe, Y. Matsui, H. Yamashita, and N. Kikuchi, Jpn. J. Appl. Phys. 31, 4205 (1992).

${ }^{3}$ R. Kumar, T. Ohta, and Y. Yamashita, Jpn. J. Appl. Phys. 31, 4195 (1992)

${ }^{4}$ G. M. Wells, H. T. H. Hector, R. L. Engelstad, and S. R. Palmer, Proc. SPIE 1465, 124 (1991).

${ }^{5}$ C. Goldsmith, T.-H. Lin, B. Powers, W.-R. Wu, and B. Novell, IEEE Microwave Theory Tech. Symp. Digest 1, 91 (1995).

${ }^{6}$ C. Y. Chi and G. M. Rebeiz, IEEE Microwave Theory Tech. Symp. Digest 43, 730 (1995).

${ }^{7}$ T. M. Weller, L. P. Katehi, M. I. Herman, and P. D. Wamhof, IEEE Microwave Theory Tech. Symp. Digest 2, 911 (1994).

${ }^{8}$ S. V. Robertson, L. P. Katehi, and G. M. Rebeiz, IEEE Microwave Theory Tech. Symp. Digest 2, 625 (1994).

${ }^{9}$ T. M. Weller, L. P. Katehi, and G. M. Rebeiz, IEEE Microwave Guided Wave Lett. 5, 153 (1995).

${ }^{10}$ A. Fartash, I. K. Schuller, and M. Grimsditch, J. Appl. Phys. 71, 4244 (1992).

${ }^{11}$ T. Tsakalos, Thin Solid Films 75, 293 (1981). 
${ }^{12}$ O. Tabata, K. Kawahata, S. Sugiyama, and I. Igarashi, Sensors Actuator 20, 135 (1989).

${ }^{13}$ B. S. Berry, W. C. Pritchet, and C. E. Uzoh, J. Vac. Sci. Technol. B 7, 1565 (1989).

${ }^{14}$ M. A. Maden, A. Jagota, S. Mazur, and R. J. Farris, J. Am. Ceram. Soc. 77, 625 (1994)
${ }^{15}$ Q. K. Tong, M. A. Maden, A. Jagota, and R. J. Farris, J. Am. Ceram. Soc. 77, 636 (1994).

${ }^{16}$ L. F. Kinsler, A. R. Frey, A. B. Coppens, and J. V. Sanders, Fundamentals of Acoustics, 3rd ed. (Wiley, New York, 1982), p. 78.

${ }^{17}$ P. M. Morse and K. Uno Ingard, Theoretical Acoustics (McGraw-Hill, New York, 1968), p. 91. 